\title{
Childhood Hyperuricemia as Risk Factor of Hypertension in Adulthood
}

\author{
Oke Rina Ramayani ${ }^{1 *}$ \\ ${ }^{1}$ Department of Pediatrics, Faculty of Medicine, University of Sumatera Utara, \\ JI.Dr. Mansyur No.5, Medan, Indonesia \\ *Correspondence: oke_rina@yahoo.com
}

\section{Abstract}

Uric acid is the end product of purine metabolism. Hyperuricemia can occur because of decreased excretion, increased production and/or a combination of both mechanisms. Elevation of uric acid in the blood $(>5.5$ $\mathrm{mg} / \mathrm{dL}$ ) in children is associated with the occurrence of essential hypertension. The relevance of pediatric hyperuricemia into adult hypertension have been widely studied.

The high percentage of children and adolescents with metabolic syndrome who had an elevated concentration of uric acid could be of great concern if it were concluded that uric acid was an independent risk factor for cardiovascular disease. The minimum age that has shown blood pressure is significantly associated with adult life is unknown. There are a number of possible explanations for the phenomenon of blood pressure tracking, including hyperuricemia. Several pathophysiological mechanisms increase uric acid with cardiovascular damage through proliferation of vascular smooth muscle cells, stimulate inflammatory path, and then prothrombotic effects triggered by the activation of platelets. Once vascular lesion has appeared, then arises the sodium-sensitive hypertension, although uric acid levels have returned to normal. Persistant mechanism of sodium sensitivity is caused by renal ischemia that leads to activation of the renin-angiotensin system, renal vasoconstriction and increased reabsorption of salt. This supports better understanding of the link between childhood hyperuricemia and adulthood hypertension.
Childhood hyperuricemia is an independent risk factor of hypertension and is 'linked to' adult blood pressure.

KEYWORDS: Uric Acid, Hyperuricemia, Primary Hypertension, children, adult

Indones Biomed J 2012; 4 (1): 12-16

\section{Introduction}

Hypertension is a major public health issue affecting approximately $20 \%$ of adults. The prevalence increases in accordance with age. Hypertension significantly increases the risk of coronary heart disease, stroke, congestive heart disease and renal failure (1). Hypertension in children, although rare, has proven to be the root of hypertension in adults. Increased blood pressure in childhood is a very good predictor for adult hypertension. Prevention of hypertension plays an important role to prevent long term morbidity and mortality (2).

Pediatric hypertension is systolic blood pressure and/or diastolic blood pressure of an average of $\geq 95$ th percentile according to sex, age, and height on $\geq 3$ times measurements (3). Hypertension in children generally arises due to kidney disease, cardiovascular or endocrine disorders, in which $30-60 \%$ of its etiologies can be identified. The most common cause is renal parenchymal disease and endocrine and cardiovascular diseases occur less frequently. The rest with unknown underlying etiologies are diagnosed as primary hypertension (essential 
hypertension) (1). The proportion of hypertension in children by age and country varies between $2-75 \%$ (4). Children with primary hypertension are generally obese $(50 \%)$ and the hypertension is often progressive until adulthood (1).

Several clinical and laboratory researches have proven true the hypothesis that uric acid is important factor in the occurrence of primary hypertension. The relationship between gout and hypertension was first reported in 1879 . Hyperuricemia has been known as a predictor and become an independent risk factor for hypertension in adults (5). Twenty-five to $40 \%$ of adult patients with untreated hypertension have a high blood uric acid level (>6.5 mg/ $\mathrm{dL}$ ), but many have a high normal uric acid level (5 to 6.5 $\mathrm{mg} / \mathrm{dL})(6)$.

Greater interest has recently been increasing among researchers to investigate how hyperuricemia is related with hypertension in children. Another important issue is the relationship between childhood hyperuricemia and hypertension in adulthood. Feig et al. showed a significant correlation between elevated uric acid and blood pressure in children and adolescent (7). Relationship of uric acid in childhood with blood pressure in adulthood has also been studied by Alper et al. (8). The purpose of this review is to describe about uric acid metabolism and its relationship with hyperuricemia and hypertension in childhood to adulthood.

\section{Urid Acid Metabolism}

Uric acid is the end product of purine metabolism (Figure 1). Approximately two-thirds of total body uric acid is produced endogenously (especially in the liver), while the other one-third is obtained from the diet containing purine $(9,10)$. Levels of uric acid in the blood is the result of a balance between solving purine and uric acid excretion rate. Excretion uric acid is performed mainly through the kidneys and the rest through the intestinal tract. Kidney failure will cause the excretion of uric acid through intestinal to increase to compensate the decreased excretion through the kidneys (9).

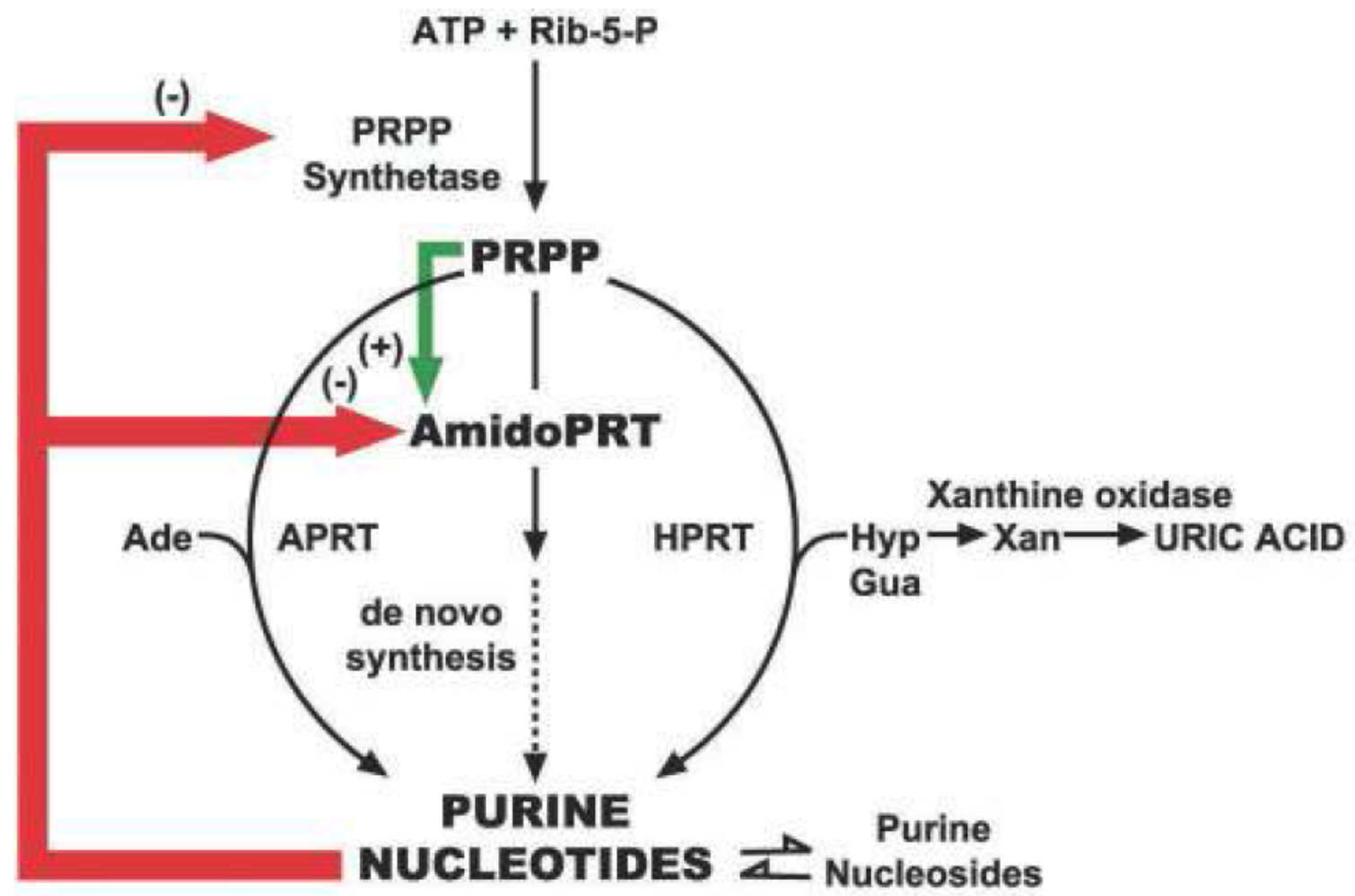

Figure 1. Purine metabolic pathway ending in the production of uric acid. (Reprint with permission from R.Terkeltaub and BioMed Central Publisher 2006).

Ade $=$ Adenine $;$ HYP = Hypoxantine; Gua = Guanine, Aprt = Adeninephosphoribosyltrasferase; HPRT = Hypoxantine-Guaninephosphoribosyltransferase. 
Uric acid in the blood is saturated at 6.4 to $6.8 \mathrm{mg} / \mathrm{dL}$, with the upper limit of solubility at levels of $7 \mathrm{mg} / \mathrm{dL}$. Uric acid is filtered at the glomerulus and then it is reabsorbed in the proximal tubule by a uric acid transporter (URAT1). Uric acid secretion correlates with plasma uric acid concentration and increased concentration in the serum leads to increased excretion of uric acid. Hyperuricemia can occur because of decreased excretion (in patients with acidosis, diuretic therapy and diabetes insipidus) and increased production (high purine diet or a high fructose diet, the increase degradation purine in leukemia, cell death in rhabdomyolisis, cytotoxic therapy) or a combination of both mechanism (9). Uric acid can accumulate in various tissues, including in the walls of blood vessels through several mechanisms of passive transudation of plasma, active transport (as in renal tubular cells), forming locally through xanthine oxidase and production of intracellular purine precursors (11).

Debate: Hyperuricemia as Risk

Factor of Cardiovascular Disease Versus as Confounding Factor

Several studies have shown that serum uric acid plays a role in the development of cardiovascular morbidity, including hypertension. A meta-analysis research that included 8 studies on hypertensive patients showed that any increase in serum uric acid resulted in an increased risk of cardiovascular diseases (12). Sanchez et al. showed that glomerular capillary pressure correlated positively with serum uric acid and systolic blood pressure (13). However, whether serum uric acid is an independent risk factor remains to be on the debate. In fact, mild hyperuricemia is often found along with obesity, lipid abnormalities, and insulin resistance, all of which are components of metabolic syndrome. After adjustment of the body mass index, age, creatinine clearance, and high density lipoprotein cholesterol, each standard deviation increase in serum uric acid entails a $75 \%$ higher risk of having cardiac hypertrophy and two times greater risk of having carotid abnormalities (14).

Several major cardiovascular risk factors associated with high serum uric acid concentrations. This mechanism is confounded interpretation relationship between serum uric acid and cardiovascular risk (15). Nevertheless, some studies still found an association between serum uric acid and cardiovascular risk despite the fact the confounding risk factors have been corrected (12-14).
Epidemiological studies in the United States on children and adolescents with metabolic syndrome have found no association between serum uric acid concentrations and the prevalence of metabolic syndrome. Although uric acid is not part of the metabolic syndrome, there is a correlation between concentrations of uric acid and metabolic syndrome or its components. Prevalence of metabolic syndrome rose from $<1 \%$ in participants with uric acid concentration $\leq 249.8 \mathrm{umoL} / \mathrm{L}(\leq 4.2 \mathrm{mg} / \mathrm{dL})$ to $21 \%$ in those with uric acid concentration $>339 \mathrm{umoL} / \mathrm{L}$ ( $>5.7 \mathrm{mg} / \mathrm{dL}$ ). The high percentage of children and adolescents with metabolic syndrome who had an elevated concentration of uric acid could be of great concern if it were concluded that uric acid was an independent risk factor for cardiovascular disease (16).

\section{Serum Uric Acid is Important in the Genesis of Primary Hypertension}

Increased consumption of fructose, which is associated with obesity in childhood, has been shown to elevate concentration of serum uric acid. Fructose is a simple sugar present in honey and fruit. Many foods or beverages such as soft drinks, candies and other sweetened products are rich in fructose. Fructose reduces endothelial nitric oxide by elevating uric acid levels. Nitric oxide deficiency causes endothelial dysfunction and insulin resistance $(17,18)$.

Feig and Johnson found that serum uric acid was significantly elevated in children with primary hypertension or secondary hypertension. A vast majority of children $(89 \%)$ with primary hypertension had serum uric acid levels above $5.5 \mathrm{mg} / \mathrm{dL}$; which is in contrast to $30 \%$ of children who had secondary hypertension, and none among children with white coat hypertension or the controls. Correlation between serum uric acid levels with systolic and diastolic blood pressure was found stronger in children than that typically observed in adults. These results support the hypothesis that uric acid might have a role in the early pathogenesis of primary hypertension (7). Results of a pilot study in children showed that uric acid may have a direct contribution to the onset of hypertension. Five children aged 14-17 years with a diagnosis of essential hypertension were treated with allopurinol only for 1 month. After one month of medication, one of the five children showed a decrease in blood pressure and the other four children had normal blood pressure. It was also found that the five children regained their higher blood pressure after discontinuation of allopurinol $(19,20)$. 
The pathophysiological factors that link increased uric acid with cardiovascular damage at the cellular and tissue levels are vascular smooth muscle cell proliferation, stimulation of inflammatory pathway, and the possible effects triggered by the prothrombotic effect mediated by thrombocyte activation (20). Uric acid has been found as mitogenic for vascular smooth muscle cell. Strong evidences have been found that uric acid activates cell growth via specific intracellular signaling events (11). Once vascular changes occur, the hypertension will be governed by the kidneys, and decrease in uric acid levels gives no protective effect on the cardiovascular disease (7).

What mechanism is involved in the event of increased serum uric acid in children with primary hypertension? At first it has been allegedly it is due to decreased renal blood flow that occurs in subjects with hypertension, which can lead to increased reabsorbtion of uric acid and elevated plasma uric acid. This argument has made some experts conclude that the increase in the level of plasma uric acid only reflects the degree of severity of the hypertension, rather than influencing the pathogenesis. However, some evidences are in existence to support that serum uric acid has some influence in the occurrence of hypertension. First, elevated uric acid has been proven as an independent predictor for hypertension (6). Second, infusion of uric acid in human subjects causes endothelial dysfunction and impaired vasodilatation. These are features of essential hypertension (20). Third, if the uric acid were simply a marker of the severity of hypertension, then it should be similarly found in secondary hypertension as well, but this has proven not to be true (7). A recent study has provided a plausible physiologic mechanism by which increase in serum uric acid might cause hypertension. Uric acid enters into vascular smooth muscle cells and stimulates the protein kinase, cyclooxygenase 2 and platelet derived growth factor, which causes vascular smooth muscle cell proliferation, macrophage infiltration and preglomerular arteriolopathy $(7,11,20)$.

Elevation of uric acid also causes platelet aggregation, which is a predisposing factor for thrombosis and atherosclerosis. Hyperuricemic patients showed a decrease in nitric oxide. Nitric oxide is liberated from the endothelium and has an effect on local cardiovascular function, including relaxation of vascular smooth muscle and vasodilatation, inhibition of platelet and leucocyte adhesion to endothelium and inhibition of myointimal hyperplasia. Loss of endothelium derived from nitric oxide bioavailability, so called 'endothelial dysfunction', is believed to be a characteristic finding in patient with several cardiovascular risk factors (15). Furthermore, increase in serum uric acid exhibits an increase in juxtaglomerular renin and a decrease in macula densa neuronal nitric oxide synthase (23). Once vascular lesion has appeared, then arises the sodium-sensitive hypertension, although uric acid levels has returned to normal (24). Persistence mechanism of sodium sensitivity is associated with renal ischemia leading to activation of the renin-angiotensin system, renal vasoconstriction.

\section{Hyperuricemia in Childhood 'Linked to' Adult Blood Pressure}

Highlights of the relevance of pediatric hypertension in adult cardiovascular health have been widely studied. The minimum age that has shown blood pressure is significantly associated with adult life is unknown. There are a number of possible explanations for the phenomenon of blood pressure tracking, including birth weight, hyperuricemia, and genetics (4).

Serum uric acid level in childhood correlates with increased systolic and diastolic blood pressure that continues until adulthood. This shows that elevation of blood uric acid level plays a role early in the development of hypertension. Alper studies have supported the assumption that if elevated levels of uric acid caused kidney damage, hypertension sensitive to sodium would occur, and this would continue even if the uric acid level had returned to normal limit (8). So maintenance of keeping uric acid level low is more effective than lowering the level of uric acid in the treatment of hypertension because if the intra-renal damage has already occurred, the hypertension induced by renal disorders will be difficult to control (24).

Increased uric acid associated with hypertension is often found in children born with low birth weight. Elevation of serum uric acid in the child can occur through genetic or environmental mechanisms. Children who are small at birth exhibit impaired endothelial function and increased systolic blood pressure and uric acid level. Low birth weight has been associated with a reduced number of nephrons and lack in maturation of the renin angiotensin system. Increased reabsorption which may stimulate the reabsorption of uric acid is a consequence of low birth weight (25). Therefore, hyperuricemia is associated with hypertension in children and adults. The control of uric acid by both dietary and pharmacologic approaches is becoming useful for lowering blood pressure and preventing morbidity and mortality (4). 


\section{Summary}

Childhood hyperuricemia is an independent risk factor of hypertension and is 'linked to' adult blood pressure. Elevated serum uric acid can lead to endothelial dysfunction and reduced bioavailability of endothelial nitric oxide. Once vascular changes have occurred, the hypertension will be governed by the kidneys and as such decreasing uric acid levels have no protective effect on the cardiovascular disease.

\section{References:}

1. Norwood VF. Hypertension. Pediatr Rev 2002; 23: 197-208.

2. Luma GB, Spiotta RT. Hypertension in childhood and adolescents. Am Fam Physician 2006; 73: 1158-68.

3. National High Blood Pressure Education Program Working Group on High Blood Pressure in Children and Adolescents. The fourth report on the diagnosis, evaluation, and treatment of high blood pressure in children and adolescent. Pediatrics 2004; 114: 555-73.

4. Hadstein C, Schaefer F. What adult nephrologist should know about childhood blood pressure. Nephrol Dial Transplant 2007; 22: 2119-23.

5. Fang J, Alderman MH. Serum uric acid and cardiovascular mortality. The NHANES I epidemiologic follow up study, 1971-1992. JAMA 2000; 283: 2404-10.

6. Selby JV, Friedman GD, Quesenberry CP. Precursors of essential hypertension: pulmonary function, heart rate, uric acid, serum cholesterol, and other serum chemistries. Am J Epidemiol 1990; 131: 1017-27.

7. Feig DI, Johnson RJ. Hyperuricemia in childhood primary hypertension. Hypertension 2003; 42: 247-52.

8. Alper AB, Chen W, Yau L, Srinivasan SR, Berenson GS, Hamm LL. Childhood uric acid predicts adult blood pressure: the Bogalusa Heart Study. Hypertension 2005;45:34-8.

9. Qazi Y. Hyperuricemia. Available from: http://www.emedicine. com/med/TOPIC1112.htm. Download May 27th 2008.

10. Terkeltaub R, Bushinsky DA, Becker MA. Recent developments in our understanding of the renal basis of hyperuricemia and the development of novel antihyperuricemic therapeutics. Arthr Res Ther 2006; 8: 1-9.

11. Rao GN, Corson MA, Berk BC. Uric acid stimulates smooth muscle cell proliferation by increasing platelet derived growth factor A-chain expression. J Biol Chem 1991; 266: 8804-8.
12. Gueyffier F, Boissel JP, Pocock S, Boutitie F, Coope J, Cutler J, et al. Identification of risk factors in hypertensive patients: contribution of randomized controlled trials through an individual patient database. Circulation 1999; 100: e88-94.

13. Sanchez-Losada LG, Tapia E, Avila-Casao C, Soto V, Franco M et al. Mild hyperuricemia induces glomerular hypertension in normal rats. Am J Physiol Renal Physiol 2002; 283: F1105-10.

14. Viazzi F, Parodi D, Leoncini G, Parodi A, Falqui V, Ratto E et al. Serum uric acid and target organ damage in primary hypertension. Hypertension 2005; 45: 991-6.

15. Waring WS, Esmail S. How should serum uric acid concentrations be interpreted in patients with hypertension? Curr Hypertens Rev 2005; 1: 89-95.

16. Ford ES, Chaoyang L, Cook S, Choi HK. Serum concentrations of uric acid and the metabolic syndrome among US children and adolescent. Circulation 2007; 115: 2526-32.

17. Heinig M, Johnson RJ. Role of uric acid in hypertension, renal disease, and metabolic syndrome. Cleveland Clinic J Med 2006; 73: 1059-64.

18. Nakagawa T, Tuttle KR, Short RA, Johnson RJ, Hypothesis: fructose induced hyperuricemia as a causal mechanism for the epidemic of the metabolic syndrome. Nat Clin Practice 2005; 1: 80-6.

19. Feig DI, Nakagawa T, Karumanchi SA, Oliver WJ, Kang DH, Finch J, Johnson RJ. Hypothesis: uric acid, nephron number and the pathogenesis of essential hypertension. Kidney Int 2004; 66: 281-7.

20. Feig DI, Mazzali M, Kang DH, Nakagawa T, Price K, Kannelis J, Johnson RJ. Serum uric acid; a risk factor and a target for treatment? J Am Soc Nephrol 2006; 17: S69-73.

21. Wang JG, Staessen JA, Fagard RH, Birkenhagen WH, Gong L, Liu L. Prognostic significance of serum creatinine uric acid in older Chinese patients with isolated systolic hypertension. Hypertension 2001; 37: 1069-74.

22. Johnson RJ, Herrera-Acosta J, Schreiner GF, Rodriguez-turbe. Subtle acquired renal injury as a mechanism of salt sensitive hypertension. N Engl J Med 2002; 346: 913-23.

23. Mazzali M, Hughes J, Kim YG, Jefferson A, Kang DH, Gordon KL, Lan HY, Kivlighn S, Johnson RJ. Elevated uric acid increases blood pressure in the rat by novel crystalindependent mechanism. Hypertension 2001; 38: 1101-6.

24. Watanabe S, Kang DH, Feng L, Nakagawa T, Kanellis J, Lan $H$, Mazzali M, Johnson RJ. Uric acid, hominoid evolution and pathogenesis of salt sensitivity. Hypertension 2002; 40: 355-60.

25. Franco MCP, Christofalo DMJ, Sawaya AL,Ajzen SA, Sesso R. Effects of low birth weight in 8-13 year old children: implication in endothelial function and uric acid level. Hypertension 2006; 48: 45-50. 